\title{
STUDY ON FOREST VEGETATION CLASSIFICATION BASED ON MULTI- TEMPORAL REMOTE SENSING IMAGES
}

\author{
Xia Jing ${ }^{1,2}$, JiHua Wang $^{2}$, WenJiang Huang ${ }^{2, *}$, LiangYun Liu ${ }^{2}$, JinDi \\ Wang ${ }^{1}$ \\ ${ }^{1}$ School of Geography, Beijing Normal University, Beijing 100875, china \\ ${ }^{2}$ National Engineering Research Center for Information Technology in Agriculture, Beijing \\ 100097, china \\ * Corresponding author, Address: National Engineering Research Center for Information \\ Technology in Agriculture, P.O.Box 2449-26,Beijing, 100097, P.R.China, Tel: +86-10- \\ 51503647(0),Fax:+86-10-51503750,E-mail:yellowstar0618@163.com; huangwj@ \\ nercita.org.cn
}

Abstract: It is very difficult to classify forest vegetation in mountain areas because of the impact of complex terrain. A new method, classification of forest vegetation based on multi-temporal remote sensing, is proposed in this paper. The forest vegetation could get better classification precision by avoiding the interactions of different plants with multi-temporal images. So it enhanced the separability of coniferous forest and broadleaf forest. The classification result showed that the accuracy could be greatly improved by using multi-temporal remote sensing images. The overall accuracy and kappa coefficient were $81.3 \%$ and 0.72 , respectively. So the method delivered in this essay has obviously technological advantages and important application potentiality in forest vegetation classification.

Keywords: multi-temporal, remote sensing, forest vegetation, classification

\section{INTRODUCTION}

The forest classification is an important aspect of the remote sensing application. Due to complexity of mountain topography, it was always a difficult problem to classify vegetation type, especially in mountain areas. In

Please use the following format when citing this chapter:

Jing, X., Wang, J., Huang, W., Liu, L. and Wang, J., 2009, in IFIP International Federation for Information Processing, Volume 293, Computer and Computing Technologies in Agriculture II, Volume 1, eds. D. Li, Z. Chunjiang, (Boston: Springer), pp. 115-123. 
order to improve classification accuracy, some classification methods were studied by many experts and scholars at home and abroad in recent years. Chen, Y.H. et al. presented a synthetic approach to automatically derive classification knowledge with purpose of constructing a model of decision tree for final classification of the image. The result showed that the approach was far superior to the traditional maximum likelihood classification method (Chen, et al., 2006). Vegetation index was proposed to classify plant types. Yuan, J.G. thought that PVI could easily identify broadleaf forest and conifer forest, and the RVI difference was obvious in different coniferous forest species (Yuan, 1999). Wang, R.H. et al. studied the forest vegetation classification of TM image with the error back propagation (BP) model. The elevation channel, combining with the three multi-spectral channels, could improve the classification accuracy. Compared with the accuracy of the maximum likelihood classifier, the classification accuracy of spruce, pine and larch with error back propagation model was increased by $27.5 \%$ (Wang, et al., 2003). According to the relations between plant type and geographical environment factor, a knowledge-based vegetation classification was presented. Compared to the supervised classification method that does not use knowledge, the classification accuracy was increased about $9 \%$ using the knowledge-based method (Liu, et al., 1998). Mo, Y.F. et al. proposed a new method, sub-region classification, to deal with the remote sensing image classification in mountain areas. The result showed that the overall accuracy of sunshine classification was up to $83.16 \%$ and the shadow classification arrived at $64.45 \%$ (Mo, et al., 2000). In order to improve the vegetation classification accuracy of Landsat TM data in mountain area, vegetation classification supported by GIS data and phonological information is made by using four-seasonal TM data of maocer. The overall accuracy is increased by $19.6 \%$ compared to the classification result of single seasonal data (Huang, et al., 2004). Three-layer BP neural network was applied to study on wetland vegetation classification in cukurova Delta distinct. The classification overall accuracy was increased from $76 \%$ to $90.2 \%$ (Berberoglu, et al., 2004). Feature extraction and forest classification were completed using some methods such as fuzzy clustering method, stepwise regression and principal component analysis based on SAR data. The result showed that the classification accuracy was higher at $71.11 \%$ using fuzzy clustering method (Wu, et al., 2000).

These methods can improve the classification accuracy to some extent. The classification of remote sensing image mainly depends on the gray value difference of plants. Because of the phenomenon that the different plants with the similar spectral and the same plants with different spectral, it is hard to classify plant types using single temporal image. Especially in the mountain areas, the problem, the different plants with the similar spectral and the same plants with different spectral, is more serious because of 
complex topography. Moreover, before classifying mountain plants, the fusion of remote sensing image and DEM data not only can correct the topography distortion, but also can improve the classification accuracy. In view of this, multi-temporal classification was proposed in mountain areas. Firstly, in order to correct the image distortion that was caused by topographic relief, Topographic correction to TM image was completed using DEM data. So the plant spectral difference which is caused by various terrain conditions can be reduced. Secondly, multi-temporal remote sensing data can fully use the time effect of spectrum signature among different plants. This technology could avoid the problem of "different spectrum with the same feature" and "different feature with the same spectrum" in some regions. The forest vegetation could get better classification precision by avoiding the interactions of different plants with multi-temporal images.

\section{MATERIALS AND METHODS}

\subsection{Study area}

Miyun is located in the northeast of Beijing (N40 $13^{\prime} 7^{\prime \prime}-40^{\circ} 47^{\prime} 57^{\prime \prime}$, E116 $\left.39^{\prime} 33^{\prime \prime}-117^{\circ} 30^{\prime} 25^{\prime \prime}\right)$, with the annual mean temperature at $10.8^{\circ} \mathrm{C}$. MiYun is the biggest county of land area in Beijing. Its total area is 2229.45 $\mathrm{km} 2$, which covers $13 \%$ of the whole Beijing city. The mountain area is $1771.75 \mathrm{~km} 2$, accounting for about $79.5 \%$ of the whole county's area. The forest coverage rate is $47.33 \%$. It is warm temperate continental climate of semi-dry and semi-humid. Its major vegetation type is warm-temperate deciduous broad-leaved forest and temperate coniferous forest.

\subsection{Data pre-processing}

It is necessary to preprocess the remote sensing data and terrain factor before forest vegetation classification with remote sensing. The main content of remote sensing data preprocessing are as follows: geometric correction, topographic correction, atmospheric correction and tasseled cap transform and so on. The terrain factor preprocessing is mainly acquisition of terrain factor using topographic map.

\subsection{Image preprocessing}

Considering image quality, temporal and spatial resolution, two TM images were acquired on April 1, 2004 and June 23, 2005 in order to classify 
plant types in Miyun mountain areas. The two images were very clear without cloud or mist.

\section{$2.4 \quad$ Geometric correction}

The Landsat TM/MSS images were geometrically corrected using bilinear interpolation based on 1:1000 digital raster graphs, and the geometric precision was superior to one pixel.

\subsection{Topographic correction}

Due to the topographic effects in the mountain, the shadow of remote sensing image is especially serious. So it is needed to correct topographic effect. The topographic correction was achieved using moment matching algorithm in this paper. Digital elevation model (DEM) was selected to obtain the slope and aspect information, and the digital number (DN) values of the image in different slope and aspect ranges were corrected to the pixels in a referenced slope and aspect range (Zhong, et al., 2006).

\subsection{Atmospheric correction}

Firstly, the Empirical Line (EL) calibration method was employed for atmospheric correction to the TM image on July 23, 2005, and the TM image was converted from digital number to reflectance (Farrand, 1994). Secondly, the pseudo invariant objects, such as water, bare soil, dam, dense vegetation, were selected from the two Landsat TM/MSS images. Finally, the MSS image on July 14, 1979 was matched to the TM image on July 23, 2005 using the least squares regression method, and the MSS image was also converted from DN value to reflectance (Schott, 1988).

\subsection{Normalized Different Vegetation Index}

Normalized Different Vegetation Index $(N D V I)$ is one of the most widely used in many vegetation indexes. It is the best indicator factor to reflect the crop condition and spatial distribution (Zhao, 2003). According to the characteristics that chlorophyll can significantly assimilate energy in red band and near infrared is sensitive to the difference of various green plants. NDVI is calculated by bands combination of red and near infrared. The formula is as follows: 


$$
N D V I=\frac{N I R-R E D}{N I R+R E D}
$$

Where NIR is reflectivity of near infrared and $R E D$ is reflectivity of red band.

\subsection{Tasseled cap transform}

Tasseled cap transform is the effective separation of plant and soil by linear variable and spectral space rotation in the multi-dimensional spectral space. TC1 describes "soil lightness" and reflects soil spectrum information; TC2 denotes "greenness" and represents vegetation spectral information (Zhao, 2005). TM greenness of Miyun on June 23, 2005 was illustrated as fig.1.

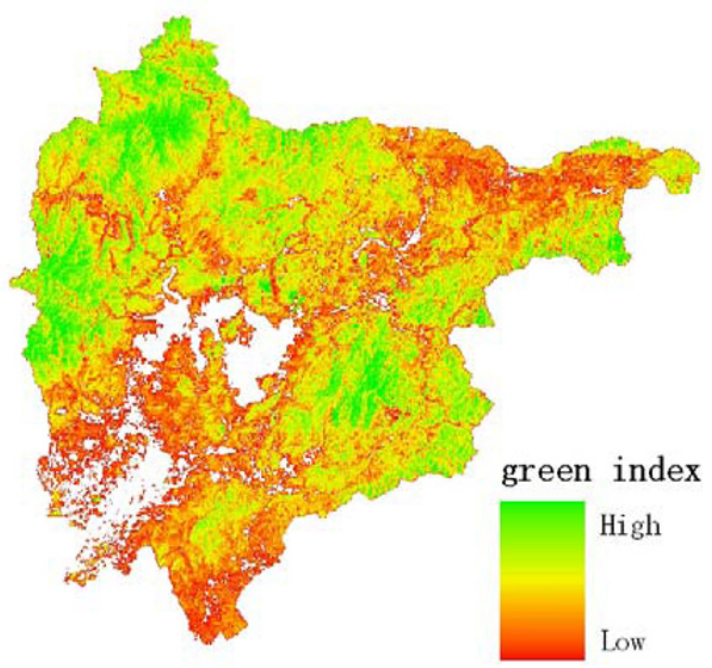

Fig. 1 TM greenness on June 23, 2005

\subsection{DEM data}

The DEM data with a spatial resolution of $30 \mathrm{~m}$ was converted from the digital contours of Miyun District at scale of 1:10000. Firstly, 1:10000 topography map was translated to digital image by scanner. Secondly, the vector contours was extracted from raster image by means of digital method. Finally, DEM data was obtained using interpolation of contours. 


\section{RESULTS}

Considering the complexity of mountain terrain, it was advanced that a new classification method based on multi-temporal remote sensing data to classify plant type in mountain area. Comparing with the single temporal data, the multi-temporal data has obvious advantages in vegetation classification because it can reflect the change of the different season vegetation spectrum characteristics. Different plant types were classified using decision tree algorithm based on the combination multi-temporal data and terrain data (Technology roadmap of classifying plant types was illustrated as fig.2).

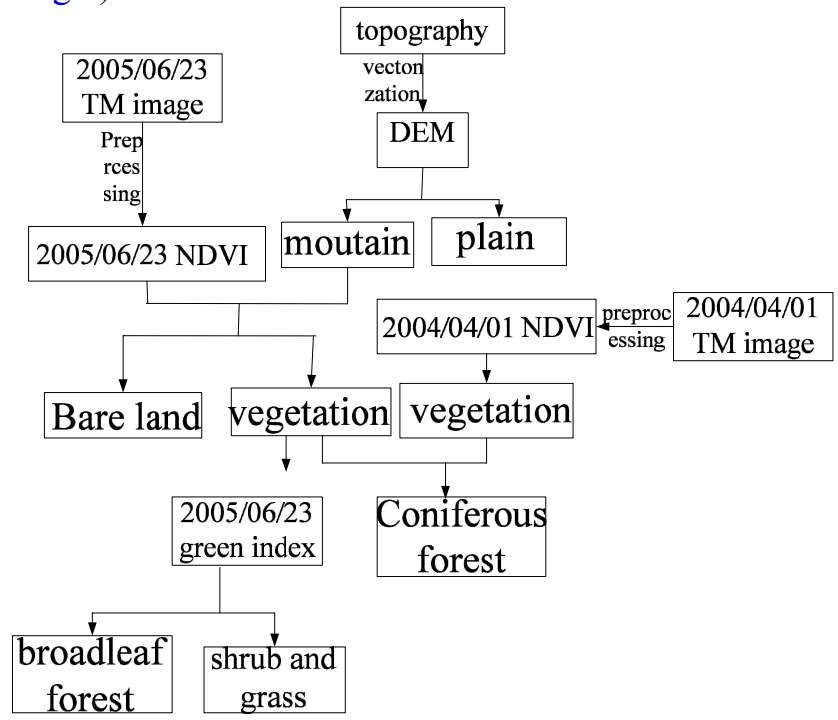

Fig.2 Technology roadmap of classifying plant types

First of all, the study area was divided into mountain and plain area. According to the actual situation in mountain areas of Miyun, the separatrix of mountain and plain area is 180 meters, namely, the region is mountain if DEM data is more than 180 meters, and otherwise, it is plain area. Secondly, in order to classify vegetation and nonvegetation, the right threshold was selected from NDVI image on June 23, 2005. Statistical analysis on the NDVI image on June 23, 2005 showed that vegetation and nonvegetation could be classified on the threshold of 0.3 . On this basis, the different plant types in Miyun mountain areas were classified using NDVI and greenness on June 23, 2005 and April 1, 2004. The main plant types in Beijing are warmtemperate deciduous broad-leaved forest and temperate coniferous forest. Moreover, temperate coniferous forest has the evergreen characteristics. So 
coniferous forest in Miyun mountain areas can be extracted using NDVI image on April 1, 2004. Thirdly, field investigation data of GPS was transferred to NDVI image on April 1, 2004. In order to extracted coniferous forest, the best threshold was selected by statistical analysis on NDVI value on April 1, 2004. The author found that extraction precision was higher with the threshold at 0.23 . Finally, classification results were built mask, which was applied to greenness image on June 23, 2005. The broadleaf forest and shrub and grass were classified by selecting suitable threshold on the masked greenness image.

Table 1. area statistics of different plant types

\begin{tabular}{ccc}
\hline Plant type & Ha & Area percent $(\%)$ \\
\hline Broadleaf forest & 67802.22 & 44.66 \\
Coniferous forest & 35452.62 & 23.35 \\
Shrub and grass & 48576.96 & 31.99 \\
Total & 151831.8 & 100 \\
\hline
\end{tabular}

According to the theory that different plant type has different range of NDVI value and greenness index, the plant types of Miyun mountain areas, such as coniferous forest, broadleaf forest and shrub and grass, were extracted using NDVI image on April 1, 2004 and TM greenness image on June 23, 2005 (spatial distribution of different plant types was illustrated as fig. 3 and area statistics was listed as table 1).

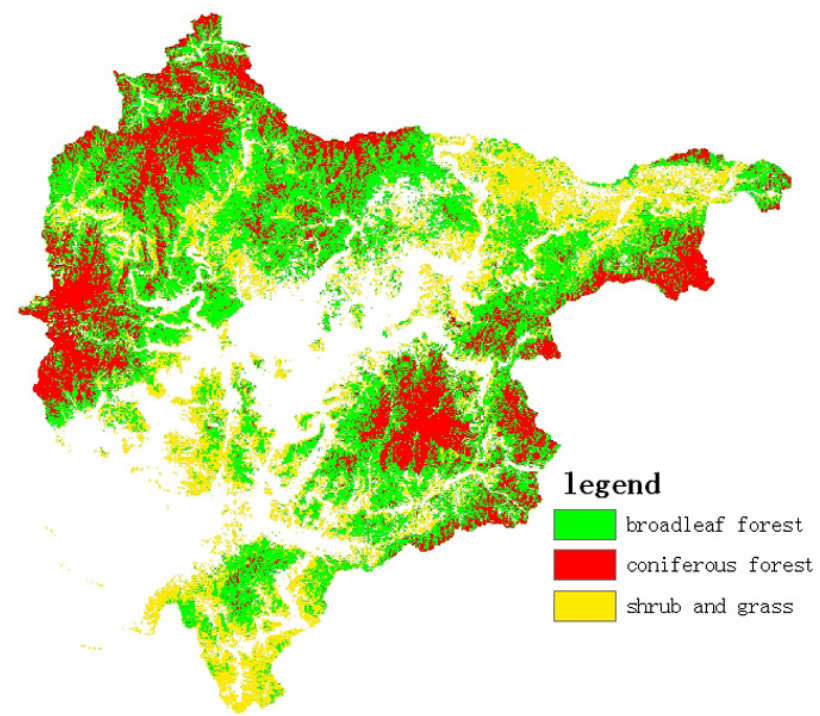

Fig. 3 spatial distribution of different plant types 
Classification result based on remote sensing image was verified using field investigation data. Accuracy analysis was completed by means of confusion matrix. Some accuracy estimating indexes, such as overall accuracy, user's accuracy, producer's accuracy and kappa coefficient, were calculated in the paper (precision estimation of forest vegetation classification was listed as table 2.).

Table2. precision estimation of forest vegetation classification

\begin{tabular}{lccccc}
\hline \multicolumn{1}{c}{ Category } & Coniferous forest & Broadleaf forest & Shrub and grass & Total & User.acc(\%) \\
\hline Coniferous forest & 128 & 15 & 12 & 155 & 82.6 \\
Broadleaf forest & 14 & 122 & 16 & 152 & 80.3 \\
Shrub and grass & 9 & 19 & 120 & 148 & 81.1 \\
Total & 151 & 156 & 148 & 455 & \\
Prod.acc(\%) & 84.8 & 78.2 & 81.1 & & \\
Overall accuracy(\%) & 81.3 & \multicolumn{5}{c}{0.72} \\
\hline
\end{tabular}

\section{CONCLUSION AND DISCUSSION}

Taking plants classification of Miyun mountain areas as an example, the feasibility, which plants classification based on multi-temporal remote sensing image, was researched in the paper. The forest vegetation could get better classification precision by avoiding the interactions of different plants with multi-temporal images. So it was enhanced that the separability of coniferous forest and broadleaf forest. The result showed that classification accuracy could be greatly improved using multi-temporal remote sensing image. The overall accuracy and kappa coefficient was $81.3 \%$ and 0.72 , respectively.

Compared with classification of single temporal image, multi-temporal remote sensing data can fully used the temporal information. Moreover ,topographic factor is closely related to the vegetation distribution in mountain areas. Classification accuracy can be improved after comprehensively considering the effect of temporal information and topographic factor on plants classification in mountain areas.

Classification based on multi-temporal remote sensing images can improve precision to forest vegetation with distinct temporal difference. Some forest vegetations, such as broadleaf forest and shrub and grass, are deciduous in winter and green leaf in summer, the advantage of multitemporal images classification can't be embodied. It needs further researched that how to improve classification precision of broadleaf forest and shrub and grass. 


\section{ACKNOWLEDGEMENTS}

The authors gratefully acknowledge the financial support provided for this research by Beijing Natural Science Foundation,(407100,), the National High Tech R\&D Program of China (2006AA10Z271, 2007AA10Z201), and National Natural Science Foundation of China (40701120) foundation of (2006BAD10A01) and foundation from Ministry of Agriculture (2006-G63).

\section{REFERENCES}

Berberoglu, S., Yilmaz, K.T, and Ozkan, C. Mapping and monitoring of coastal wetlands of Cukurova Delta in the Eastern Mediterranean region, Biodiversity and Conservation, 2004,13, 615-633.

Chen, Y.H., and Zhang, W.C. GIS supported decision tree classification of remote sensing images in mountainous area, Remote sensing for land \& resources, 2006, 67, 69-74.

Farrand, W.H., Singer, R.B., and Merenyi, E. Retrieval of Apparent Surface Reflectance from Aviris Data-a Comparison of Empirical Line, Radiative Transfer, and Spectral Mixture Methods, Remote Sens.Environ, 1994, 47,311-321.

Forest cover types using SAR imagery, International Journal of Remote Sensing, 2000, 21,2089-2099.

Huang, J.W., and Ju, H.B. Study on Integrated Vegetation Classification by Using Multitemporal TM Data in Mountainous Area of Northeast China, Journal of Fujian College of Forestry, 2004, 24, 136-139.

Liu, W.G., Gong, J.H., and Fang, H.L. Knowledge extraction from GIS database and its application in vegetation classification, Journal of remote sensing, 1998, 2, 234-240.

Mo, Y.F., and Zhou, L.X. Sub-region classification method-an new classification method to remote sensing image in mountain areas, Carsologica sinca, 2000, 19, 360-365.

Schott, J., Salvaggio, R. C., and Volchok, W.J. Radiometric scene normalization using pseudoinvariant features, Remote Sensing Environ., 1988, 26,1-16.

Wang, R.H., Huo, H.T., and You, X.X. Forest vegetation classification of TM images using artificial neural network, Journal of Beijing Forestry University, 2003, 25, 1-5.

$\mathrm{Wu}, \mathrm{D}$., and Linders, J. Comparison of three methods to select feature for discriminating

Yuan, J.G. Study of forest vegetation classification with remote sensing, Journal of Hebei Normal University(Natural Science), 1999, 23,274-278.

Zhao, Y.S. A study on environmental change analysis in Sand Hill of Nebraska using remote sensing, Geographical Research, 2005, 20,213-219.

Zhao, Y.S. The Remote Sensing Theory and Method of Application and Analysis, Beijing:Science Press, 2003, 374-393

Zhong, Y.W., Liu, L.Y., Wang, J.H., and Yan, G.J. A Method Based on Moment Matching Algorithm to Correct Remotely Sensed Image in Rugged Area,Geography and GeoInformation Science, 2006, 22,31-34. 